\title{
Overexpression of the $\beta 2 A R$ gene improves function and re-endothelialization capacity of EPCs after arterial injury in nude mice
}

Xiao Ke $\mathrm{Ke}^{1,2+}$, Xiao-Rong Shu ${ }^{1,2+}$, Fang $\mathrm{Wu}^{3}$, Qing-Song Hu ${ }^{1,2}$, Bing-Qing Deng ${ }^{1,2}$, Jing-Feng Wang ${ }^{1,2}$ and Ru-Qiong $\mathrm{Nie}^{1,2^{*}}$

\begin{abstract}
Background: Proliferation and migration of endothelial progenitor cells (EPCs) play important roles in restoring vascular injuries. $\beta 2$ adrenergic receptors ( $\beta 2 A R s)$ are widely expressed in many tissues and have a beneficial impact on EPCs regulating neoangiogenesis. The aim of the present study was to determine the effect of overexpressing $\beta 2 A R s$ in infused peripheral blood (PB)-derived EPCs on the re-endothelialization in injured vessels.

Methods: Induction of endothelial injury was performed in male nude mice that were subjected to wire-mediated injury to the carotid artery. Human PB-derived EPCs were transfected with an adenovirus serotype 5 vector expressing $\beta 2 A R$ (Ad5/32AR-EPCs) and were examined $48 \mathrm{~h}$ later. $\beta 2 A R$ gene expression in EPCs was detected by real-time polymerase chain reaction and Western blot analysis. In vitro, the proliferation, migration, adhesion, and nitric oxide production of Ad5/32AR-EPCs were measured. Meanwhile, phosphorylated Akt and endothelial nitric oxide synthase (eNOS), which are downstream of $\beta 2 A R$ signaling, were also elevated. In an in vivo study, CM-Dil-labeled EPCs were injected intravenously into mice subjected to carotid injury. After 3 days, cells recruited to the injury sites were detected by fluorescent microscopy, and the re-endothelialization was assessed by Evans blue dye.

Results: In vitro, $\beta 2 A R$ overexpression augmented EPC proliferation, migration, and nitric oxide production and enhanced EPC adhesion to endothelial cell monolayers. In vivo, when cell tracking was used, the number of recruited CM-Dil-labeled EPCs was significantly higher in the injured zone in mice transfused with Ad5/B2AR-EPCs compared with non-transfected EPCs. The degree of re-endothelialization was also higher in the mice transfused with Ad5/ $32 A R-E P C s$ compared with non-transfected EPCs. We also found that the phosphorylation of Akt and eNOS was increased in Ad5/ß2AR-EPCs. Preincubation with $\beta 2 A R$ inhibitor (ICl118,551), Akt inhibitor (ly294002), or eNOS inhibitor (L-NAME) significantly attenuated the enhanced in vitro function and in vivo re-endothelialization capacity of EPCs induced by B2AR overexpression.
\end{abstract}

Conclusions: The present study demonstrates that $\beta 2 A R$ overexpression enhances EPC functions in vitro and enhances the vascular repair abilities of EPCs in vivo via the $\beta 2 A R / A k t / e N O S$ pathway. Upregulation of $\beta 2 A R$ gene expression through gene transfer may be a novel therapeutic target for endothelial repair.

Keywords: $\beta 2 A R$, Re-endothelialization, Proliferation, Migration, Endothelial progenitor cells, $\beta 2 A R / A k t / e N O S$

\footnotetext{
* Correspondence: nieruqiong@163.com

${ }^{\dagger}$ Equal contributors

'Department of Cardiology, Sun Yat-sen Memorial Hospital of Sun Yat-sen

University, No. 107, Yanjiangxi Road, Guangzhou, China

${ }^{2}$ Guangdong Province Key Laboratory of Arrhythmia and Electrophysiology,

Guangzhou 510120, China

Full list of author information is available at the end of the article
} 


\section{Background}

Coronary atherosclerotic heart diseases are the major cause of mortality worldwide in cardiovascular disease (CVD), yet current therapies only delay disease progression and improve lifestyle without addressing the fundamental problem of tissue loss. Several studies have shown that endothelial dysfunction is an early marker of atherosclerosis, and the activation of inflammatory processes and abnormalities in vascular homeostasis have been suggested to contribute to the development of atherosclerotic vascular disease [1]. Accumulating evidence indicates that the balance between endothelial injury and repair is a key component of atherosclerosis [2], and maintaining endothelial integrity is crucially important to preventing the initiation and development of atherosclerosis, coronary heart disease, and postangioplasty restenosis [3]. Thus, accelerated re-endothelialization might prevent the early stages of atherosclerosis and restenosis after angioplasty.

Endothelial progenitor cells (EPCs) mobilized from bone marrow into the peripheral blood (PB) have been shown to play an important role for vascular regeneration, endothelial repair, and replacement of dysfunctional endothelium by incorporating into the site of vessel injury, differentiating into endothelial cells (ECs), and releasing paracrine factors $[4,5]$. Transplantation of EPCs is currently under intensive investigation in animal models and clinical research and have become a major focus of CVD treatment to accelerate re-endothelialization [6]. However, the beneficial effects of this practice have not been observed in patients with coronary artery disease, because the reparative capacity of EPCs appears to be limited by their poor survival environment [7]. Therefore, attempts to improve the function of transplanted EPCs with gene modifications may facilitate the repair of damaged endothelia and accelerate re-endothelialization.

Various molecules may be involved in the processes by which EPCs home to and restore damaged endothelium, such as nitric oxide (NO), stromal cell-derived factor 1a (SDF-1a), and vascular endothelial growth factor (VEGF). It is widely accepted that $\beta$-adrenoceptors exist on ECs and contribute to the regulation of vasomotor tone. Moreover, it is the $\beta 2$ adrenergic receptor $(\beta 2 \mathrm{AR})$, the most abundant $\beta A R$ in the vasculature, that mediates the $\mathrm{NO}$ involved in relaxing vascular tone [8]. $\beta 2 \mathrm{ARs}, \mathrm{G}$ protein-coupled receptors, are activated by adrenergic catecholamine to promote a series of intracellular signal transduction pathways that lead to multiple cell-specific responses [9]. It has been demonstrated that $\beta 2$ ARs are strongly expressed on EPCs and also mediate the homing and neovascularization capacity of EPCs to areas of ischemia [9]. Accordingly, the $\beta 2 \mathrm{AR}$ gene may be a valuable molecular target for gene therapies that use EPCs. However, $\beta 2$ ARs have not previously been shown to accelerate vascular repair via re-endothelialization mediated by
EPCs. Thus, we sought to determine whether $\beta 2 A R$ gene transfer mediates the functional properties of EPCs during vascular injury.

In this study, we tested the therapeutic potential of $\beta 2 \mathrm{AR}$ gene transfer in EPCs by infusing transfected cells into nude mice after we induced an endothelial denudation injury. We also investigated the $\beta 2 \mathrm{AR}$-mediated Akt/ endothelial nitric oxide synthase/NO (Akt/eNOS/NO) signaling pathway that is related to both in vitro and in vivo biology of EPCs. These data demonstrate that the $\beta 2 \mathrm{AR}$ has an important role in EPC migration at the vascular injury site, and upregulating $\beta 2 \mathrm{AR}$ expression is a potential new therapeutic strategy that may improve the efficiency of EPC-induced re-endothelialization.

\section{Methods}

Ethics

The experimental research on humans in this study was performed in compliance with the Helsinki Declaration. All recruited patients consented to participate in this trial and to contribute their trial data for non-commercial purposes. The protocol of this trial was externally reviewed and approved by an anonymous independent ethical review committee to ensure that there were no serious ethical concerns. The animal procedures in this study complied with the Animal Care and Use Ethics Committees of Sun Yat-Sen University.

\section{EPC culture and characterization}

EPCs were isolated and cultured according to previously described methods [10, 11]. Briefly, PB mononuclear cells (MNCs) were isolated from healthy subjects (males from 25 to 35 years of age) by using Histopaque- 1077 density gradient centrifugation at $400 \mathrm{~g}$ for $30 \mathrm{~min}$. The collected MNCs were washed three times with phosphate-buffered saline (PBS) (Jingmei Bio Tech Co. Ltd., Shenzhen, China). After the cells were purified, the MNCs were cultured on fibronectin-coated six-well plates in endothelial basal medium-2 (EBM-2) (CC-4176; Lonza, Basel, Switzerland) supplemented with EGM-2 Bulle Kit (Lonza) and $20 \%$ fetal bovine serum (FBS) (Gibco, now part of Thermo Fisher Scientific, Waltham, MA, USA). After 4 days in culture, the non-adherent cells were abandoned. Adherent cells were cultured for 7 days and then were used for the following experiments.

EPCs were defined as cells that were dually positive when stained by using $1,1^{\prime}$-dioctadecyl-3,3,3',3' -tetramethylindocarbocyanine (DiI)-acetylated low-density lipoprotein (ac-LDL) (20 $\mu \mathrm{g} / \mathrm{ml}$; Invitrogen, Carlsbad, CA, USA) and fluorescein isothiocyanate (FITC)-labeled BS-1 lectin $(10 \mu \mathrm{g} / \mathrm{ml}$; Sigma-Aldrich, St. Louis, MO, USA). Cultured EPCs were incubated with DiI-ac-LDL for $3 \mathrm{~h}$ at $37^{\circ} \mathrm{C}$; the cells then were washed in PBS, fixed in $4 \%$ (vol/vol) paraformaldehyde (PFA) for $30 \mathrm{~min}$, and 
incubated with FITC-labeled BS-1 lectin for $1 \mathrm{~h}$. The cells were washed again and then incubated with 4',6-diamidino-2-phenylindole (DAPI), a nuclear counterstain. Double-positive cells were observed with a fluorescence microscope (×200 magnification; Olympus, Tokyo, Japan). Cells demonstrating double-positive fluorescence were identified as differentiating EPCs.

\section{Flow cytometric analysis}

The expression of endothelial marker proteins was examined in the cultured EPCs by using flow cytometric analysis with phycoerythrin (PE)-labeled monoclonal mouse anti-human antibodies recognizing CD31 (BD Pharmingen, San Diego, CA, USA), von Willebrand factor (vWF) (BD Pharmingen), kinase-insert domain receptor (KDR) (R\&D Systems, Minneapolis, MN, USA), and CD14 (BD Pharmingen). To identify the cells that expressed these surface antigens, the EPCs were incubated for $40 \mathrm{~min}$ at $4{ }^{\circ} \mathrm{C}$ in a volume of $100 \mu \mathrm{l}$ of solution containing an appropriate amount of PE-labeled antibody or corresponding IgG isotype control. At least $1 \times 10^{5}$ EPCs were acquired by using a flow cytometer (Beckman-Coulter, Fullerton, CA, USA).

\section{Immunofluorescence}

To characterize the expression of EC markers, EPCs were grown in fibronectin-coated six-well plates, and immunofluorescence analysis was performed by using rabbit polyclonal antibody against $\beta 2 A R$ (Abcam, Cambridge, MA, USA) and mouse monoclonal antibody (mAb) against eNOS (Cell Signaling Technology, Boston, MA, USA). Briefly, the cells were washed in cold PBS three times and fixed in $4 \%$ PFA for $30 \mathrm{~min}$. Then the cells were washed again with PBS three times for 5 min each and incubated in $3 \%$ bovine serum albumin (BSA) in PBS for $1 \mathrm{~h}$. The cells were incubated with primary antibodies (anti-eNOS, anti- $\beta 2 A R$ diluted 1:100 with $3 \% \mathrm{BSA}$ in PBS) at room temperature for $1 \mathrm{~h}$. After the cells were washed three times for 5 min each in PBS on a shaker, the cells were exposed to goat anti-rabbit IgG $(\mathrm{H}+\mathrm{L})$ (catalog no. A-11011; Life Technologies, Carlsbad, CA, USA) and goat anti-mouse IgG $(\mathrm{H}+\mathrm{L})$ (catalog no. A-11011; Life Technologies) secondary antibodies for $1 \mathrm{~h}$ in the dark. The cells were washed again and incubated with DAPI to stain the EPC nuclei. Images were acquired by using a fluorescence microscope (×200 magnification; Olympus).

\section{EPC gene transfer}

An adenovirus sero-type 5 (Ad5) vector expressing the human $\beta 2 A R$ gene (Ad5/ $32 \mathrm{AR}$ ) or enhanced green fluorescent protein (Ad5/EGFP) was used for gene delivery (purchased from GeneChem Company Ltd., Shanghai, China). To establish the appropriate virus concentration for adenoviral gene transfer into EPCs, the effectiveness of different multiplicities of infection (MOIs) was evaluated in accordance with the instructions of the adenovirus manufacturer. Briefly, after the EPCs were cultured for 7 days, they were transduced with Ad5/ $32 \mathrm{AR}$ and Ad5/EGFP in serum-free culture medium (MOI of approximately 500). The viruses were removed, and the cells were washed with PBS and incubated with EPC medium for another $48 \mathrm{~h}$ before subsequent experiments.

\section{Real-time polymerase chain reaction and Western blot analysis}

Total cellular RNA was isolated by using TRIzolreagent (Invitrogen). Double-stranded cDNA was synthesized by using an M-MLV Reverse Transcriptase cDNA Synthesis Kit (TaKaRa, Kusatsu, Shiga, Japan). Quantitative polymerase chain reaction (PCR) was carried out with Light Cycler 480 SYBR Green I Master Mix (Roche Diagnostics, Risch-Rotkreuz, Switzerland) in a Light Cycler 480 System. The cycling protocol for the PCR was as follows: $95{ }^{\circ} \mathrm{C}$ for $5 \mathrm{~min}$, followed by 45 cycles of $95^{\circ} \mathrm{C}$ for $10 \mathrm{~s}$, $60{ }^{\circ} \mathrm{C}$ for $10 \mathrm{~s}$, and $72{ }^{\circ} \mathrm{C}$ for $20 \mathrm{~s}$. The primers used were as follows: $32 \mathrm{AR}$ : 5'-ATGGTGTGGATTGTGTCAGG-3' (forward) and 5'-CAGGTCTCATTGGCATAGCA-3' (reverse) and glyceraldehyde 3-phosphate dehydrogenase (GAPDH): 5'-GGTGGTCTCCTCTGACTTCAACA-3' (forward) and 5'-GTTGCTGTAGCCAAATTCGTTGT-3' (reverse).

EPCs were lysed with cell lysis buffer (Cell Signaling Technology) in accordance with the instructions of the manufacturer. Cell lysates were quantified by bicinchoninic acid (BCA) methods in accordance with the instructions of the manufacturer (Sangon Biotechnology, Shanghai, China). In total, $50 \mu \mathrm{g}$ protein was subjected to SDS-PAGE and then transferred to polyvinylidene fluoride membranes. The following antibodies were used: rabbit anti- $\beta 2 A R$ antibody (1:1000; Abcam, Cambridge, MA, USA), Phospho-Akt (Ser473) rabbit mAb (1:1000; Cell Signaling Technology), PhosphoeNOS (Ser1177) rabbit mAb (1:1000; Cell Signaling Technology), eNOS (49G3) rabbit mAb (1:1000; Cell Signaling Technology), Akt (C67E7) rabbit mAb (1:1000; Cell Signaling Technology), SDF-1 antibody (1:1000; Cell Signaling Technology), CXCR4(H-118) (1:1000; Santa Cruz Biotechnology, Inc., Dallas, TX, USA), and GAPDH (14C10) rabbit mAb (1:1000; Cell Signaling Technology). Proteins were visualized with horseradish peroxidaseconjugated anti-rabbit IgG (1:5000; Cell Signaling Technology). To detect the effect of stimulation of PBderived EPCs with the selective $\beta 2 \mathrm{AR}$ agonist fenoterol (FENO) on the phosphorylation of Akt and eNOS, EPCs were pre-incubated with $10^{-8} \mathrm{M}$ FENO (Sigma-Aldrich) for $6 \mathrm{~h}$ before proteins were harvested. 


\section{EPC proliferation and NO production}

The effect of $\beta 2 A R$ gene transfer into EPCs on cell proliferation was assessed by CCK8 (Dojindo Molecular Technologies, Kumamoto, Japan). EPCs were transduced with Ad5/ $\beta 2$ AR or Ad5/EGFP, or they were not transduced (control). The EPCs were reseeded on 96-well plates. Briefly, the EPCs were seeded in 96-well plates $\left(5 \times 10^{3}\right.$ cells per well) in EBM-2 (Lonza, CC-4176) supplemented with $1 \%$ FBS for $24 \mathrm{~h}$. The medium then was replaced with $100 \mu \mathrm{l}$ of fresh medium containing $10 \mu \mathrm{l}$ of CCK8 solution, and the cells were incubated for another $2 \mathrm{~h}$. The absorbance of each well then was determined at $450 \mathrm{~nm}$ by using an Infinite F200 Multimode plate reader. We used 0.3 $\mu$ M ICI118,551 (Sigma-Aldrich), $10 \mu \mathrm{M}$ LY294002 (Calbiochem, now part of EMD Millipore, Billerica, MA, USA), and $100 \mu \mathrm{M}$ L-NAME (Calbiochem) to inhibit $\beta 2 A R$, Akt, and eNOS, respectively. The EPCs were preincubated with inhibitor for $30 \mathrm{~min}$ before FENO $\left(10^{-8} \mathrm{M}\right)$ stimulations.

NO secretion by EPCs was measured as the generation of nitrite. The cells were cultured with EBM-2 (growth factor-free) for $48 \mathrm{~h}$ after gene transfer. The supernatants were assayed to determine the level of NO by using a NO assay kit by the nitrate reductase method (Nanjing Jiancheng Institute of Biological Engineering, Nanjing, China).

\section{In vitro EPC migration assays}

EPC migration assays were performed by using a Transwell system (Corning Costar, Tewksbury, MA, USA) with $8-\mu \mathrm{m}$ polycarbonate filter inserts in 24-well plates. Briefly, a total of $2 \times 10^{4}$ EPCs were suspended in $250 \mu \mathrm{l}$ of EBM-2 medium supplemented with $1 \% \mathrm{FBS}$, and the cells were incubated in the upper chamber for $30 \mathrm{~min}$ for each group. These groups included the control group, Ad5/ $\beta 2$ AR-EPC group, and Ad5/EGFP-EPC group, which were not pretreated, and the Ad5/ß2AREPC group and Ad5/EGFP-EPC group, which were pretreated with ICI118,551, LY294002, and L-NAME. The lower compartment of the modified Boyden chamber was placed in a 24-well culture plate in which each well was filled with $500 \mu \mathrm{l}$ of EBM-2 supplemented with PBS or FENO $\left(10^{-8} \mathrm{M}\right)$. After the cells were cultured for $6 \mathrm{~h}$, the cells that had migrated into the lower chamber were stained with DAPI. The transmigrated cells were randomly counted by an independent investigator who was blinded to treatments.

\section{In vitro EPC adhesion assays}

A monolayer of human umbilical vein endothelial cells (HUVECs) was prepared $48 \mathrm{~h}$ before the assay by plating $2 \times 10^{5}$ cells in each well of a four-well plate. The HUVECs were pretreated with or without $1 \mathrm{ng} / \mathrm{ml}$ tumor necrosis factor- $\alpha$ (TNF- $\alpha$ ) (PeproTech, Rocky
Hill, NJ, USA) for $12 \mathrm{~h}$. Then $1 \times 10^{5}$ CM-DiI (CellTracker $^{\text {Tw }}$ CM-DiI, Invitrogen)-labeled EPCs was added to each well, and the cells were incubated for $3 \mathrm{~h}$ at $37^{\circ} \mathrm{C}$. The non-attached cells were gently removed with PBS, and the adherent EPCs were fixed in $4 \%$ PFA and randomly counted by an independent investigator who was blinded to treatments.

\section{Animal model and in vivo re-endothelialization assay}

The carotid artery injuries and EPC transplantation were performed by using previously described methods $[12,13]$. Male NRMInu/nu athymic nude mice (The Laboratory Animal Center of Sun Yat-sen University, Guangzhou, China) that were 6 to 8 weeks old were injected with human PB-derived EPCs. The animals were anaesthetized with ketamine $(100 \mathrm{mg} / \mathrm{kg}$ intraperitoneally) and xylazine ( $5 \mathrm{mg} / \mathrm{kg}$ intraperitoneally). The surgeries were performed by using a stereoscopic microscope. The left carotid artery was exposed via a midline incision on the ventral side of the neck. The bifurcation of the carotid artery was located, and two ligatures were placed around the external carotid artery, which then was tied off with the distal ligature. An incision hole was made between the ligatures to introduce the denudation device. A curved flexible wire $(0.35-\mathrm{mm}$ diameter) was introduced into the common carotid artery and passed over the lining of artery three times to denude the endothelium. The wire then was removed, and the external carotid artery was tied off proximal to the incision hole with the proximal ligature.

EPCs $\left(1 \times 10^{6}\right.$ cells $)$ that had been cultured for 7 days were resuspended in $100 \mu \mathrm{l}$ of pre-warmed PBS $\left(37^{\circ} \mathrm{C}\right)$ and were transplanted $3 \mathrm{~h}$ after carotid artery injury via tail vein injection with a $27-G$ needle. The same volume of PBS was injected into placebo mice as a control. Three days after carotid artery injury, endothelial regeneration was evaluated by staining denuded areas with $50 \mu \mathrm{l}$ of $5 \%$ Evans blue dye via tail vein injection. To examine the homing of transplanted EPCs to the site of the injured carotid vessel, labeled EPCs $\left(1 \times 10^{6}\right)$ were incubated with CM-DiI (Cell Tracker ${ }^{\text {Tu }}$ CM-DiI; Invitrogen) in accordance with the instructions of the manufacturer. CM-DiI-labeled EPCs incorporated in the injured vessels were quantitatively analyzed under a fluorescence microscope (Olympus BX51).

\section{Statistical analysis}

All results are expressed as the mean \pm standard error of the mean. Statistical significance was evaluated by means of Student's $t$ test or analysis of variance. A $P$ value of less than 0.01 was considered to denote statistical significance. All statistical analyses used SPSS statistical software (SPSS version 13.0; IBM Corporation, Armonk, NY, USA). 


\section{Results}

\section{Characterization of EPCs and endogenous expression of} B2AR on EPCs

Recently, EPCs were classified into two distinct types: early and late EPCs. Early EPCs appear after 5 to 7 days, and late EPCs appear after 14 to 21 days [14]. A beneficial effect on vascular repair after injury has been shown for early EPCs [15]. After 7 days of culture on fibronectin-coated plates, PB-derived EPCs had a spindle-shaped morphology. Cellar immunostaining showed that most of the adherent cells have doublepositive staining for the uptake of DiI-ac-LDL and for the binding of FITC-lectin, indicating that these cells possess the functional properties of ECs (Fig. 1a). In addition, flow cytometric analysis of EC antigens revealed that $40.82 \pm 3.98 \%$ of the adherent cells were positive for CD31, $50.69 \pm 4.76 \%$ for vWF, $96.56 \pm$ $8.76 \%$ for VEGF (VEGFR2/KDR), and $55.47 \pm 4.75 \%$ for the monocytic maker CD14 (Fig. 1b). All of these characteristics indicate that the cultured adherent cells were appropriately identified as EPCs, as previously described $[12,16]$. We performed immunocytochemistry for colocalization of $\beta 2$ ARs and the EPC marker eNOS. Fluorescence microscopy revealed that $\beta 2 \mathrm{ARs}$ were found to localize to the cell membrane of EPCs, and these results were confirmed by using a positive marker, eNOS, which is also expressed in EPCs (Fig. 1c). In addition, $\beta 2 \mathrm{AR}$ expression on the EPCs did not increase when the cells were stimulated with the selective $\beta 2 \mathrm{AR}$ agonist FENO for 6 or $12 \mathrm{~h}$, as shown in the Western blot analyses. These results are consistent with the findings of Galasso et al. (Fig. 1d) [9].

\section{Overexpression of $\beta 2 A R$ in transfected EPCs}

Adenovirus effectively mediated the transfection of the $\beta 2 \mathrm{AR}$ gene into EPCs. To upregulate the $\beta 2 \mathrm{AR}$ gene in EPCs, we transduced EPCs by using Ad5/ $32 \mathrm{AR}$ gene transfection. At $24 \mathrm{~h}$ after transfection, EGFP expression was detected by fluorescent microscopy, and the transfection efficiency was approximately $80 \%$ (data not shown). At $48 \mathrm{~h}$ after transfection, our results showed that $\beta 2 A R$ mRNA was significantly increased in the Ad5/ 32 AR-EPC group compared with the Ad5/EGFPEPC group or in controls by using real-time PCR $(9.73$ \pm 3.56 versus $0.89 \pm 0.65$ or $1.00 \pm 0.47 ; P<0.01)$. These results were further confirmed by using Western blot analysis $(0.40 \pm 0.03$ versus $0.18 \pm 0.03$ or $0.2 \pm 0.01 ; P<$ 0.01) (Fig. 2a, b).

B2AR gene transfer in EPCs upregulates cell proliferation, migration and adhesion; NO production; SDF-1/CXCR4 expression in vitro

We used CCK8 assays to examine whether overexpressing $\beta 2 A R$ affected proliferation in EPCs that were stimulated with FENO. The rate of proliferation was significantly higher in the Ad5/ $\beta 2 \mathrm{AR}$-transduced EPCs than in the Ad5/EGFP-transduced EPCs or the nontransduced EPCs (Fig. 3a). We also detected NO production by analyzing NO levels in the conditioned media at $48 \mathrm{~h}$ after $\beta 2 A R$ gene transfer. As shown in Fig. $3 \mathrm{~b}$, the level of NO production was higher in the Ad5/ $\beta 2 \mathrm{AR}$ transduced EPCs than in the Ad5/EGFP-transduced EPCs or the non-transduced EPCs.

In the migration assays, there were no differences in basal migration capacity among Ad5/ $\beta 2 \mathrm{AR}$-transduced EPCs, Ad5/EGFP-transduced EPCs, and non-transduced EPCs. However, the rate of EPC migration was significantly higher in Ad/ $\beta 2 \mathrm{AR}$-transduced EPCs that were induced by FENO stimulation than in the Ad5/EGFP transduced EPCs and non-transduced EPCs that were stimulated by FENO ( $28.2 \pm 4.2$ versus $20.2 \pm 5.5$ or 20.6 \pm 4.6; $P<0.01$ ) (Fig. 3c-e).

TNF- $\alpha$ can enhance the expression of adhesion molecules in ECs [16]. To determine the function of $\beta 2 A R$ in EPC adhesion, we investigated its role in the adhesion of EPCs cultured on mature HUVEC monolayers. Similar to the results of the migration assay, without TNF- $\alpha$ stimulation, there was no difference in the adhesiveness of EPCs cultured on HUVECs among the Ad5/ $\beta 2 \mathrm{AR}$ transduced, Ad5/EGFP-transduced, and non-transduced EPC groups. However, when the HUVECs were activated with TNF- $\alpha$, the adhesive ability of the Ad5/ $\beta 2$ AR-transduced EPCs was greater than the adhesive ability of the Ad5/EGFP-transduced EPCs and the non-transduced EPCs $(61.2 \pm 7.2$ versus $27.4 \pm 4.1$ or $28.6 \pm 4.9 ; P<0.01)$ (Fig. 3d-f). Therefore, these data suggested that $\beta 2 A R$ mediated the biological function of EPCs in vitro and that $\beta 2 \mathrm{AR}$ overexpression would increase the regulatory capacity of EPCs.

The chemokine stromal-derived factor (SDF-1) and its unique receptor CXCR4 (SDF-1 and CXCR4 axis) play an important role invasculogenesis, neovascularization, and re-endothelialization $[17,18]$. SDF-1 and CXCR4 are also involved in the biological functions of EPCs, including migration, adhesion, mobilization, and homing. Interestingly, in our study, Western blot analysis revealed that SDF-1 and CXCR4 levels were significantly higher at both time points in the cells that were stimulated with FENO than in the control EPCs that were not stimulated with FENO, and this difference was stronger in the Ad5/ $32 \mathrm{AR}$-transduced EPC group (Fig. 3g). These results suggest that $\beta 2 A R$ stimulation promotes the expression of members of the SDF-1/CXCR4 axis in EPCs.

\section{$\beta 2 A R$ gene transfer increases the re-endothelialization capacity of EPCs in vivo}

The carotid endothelium injury in mice was confirmed by Evans blue staining (Fig. 4a). To assess the capability 


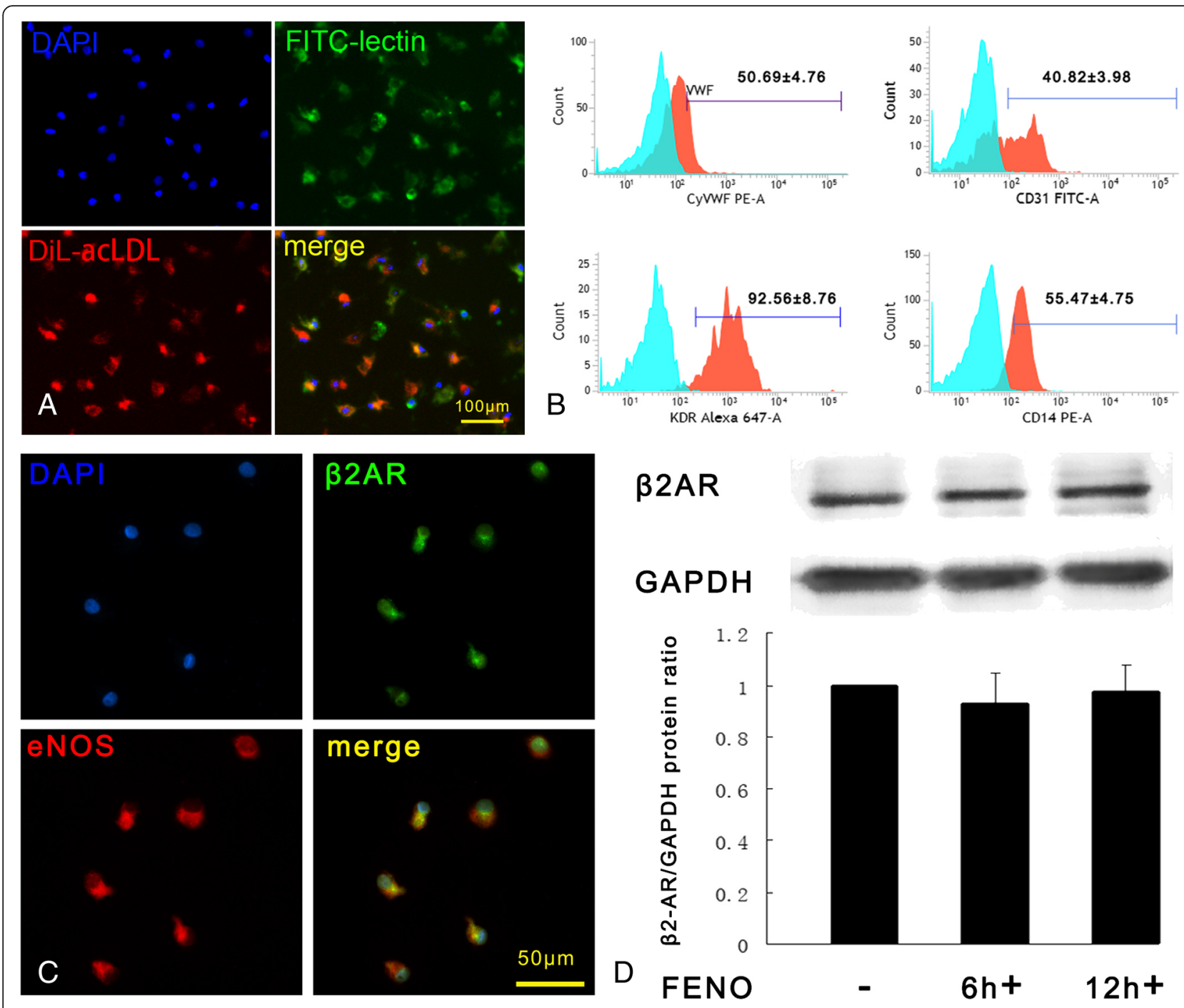

Fig. 1 Characterization of EPCs and endogenous expression of $\beta 2 A R$ on EPCs. a Representative photographs of EPCs at 7 days that were labeled with DAPI (blue), FITC-labeled BS-1 lectin (green), and Dil-acLDL uptake (red). Double-labeled cells were identified as EPCs (yellow) (x200). b Cells were tested for the ability to express the endothelial markers CD31, VWF, KDR, and CD14 by using flow cytometry analysis (IgG isotype control is shown in blue, $n=4$ per group). c The expression of $\beta 2 A R$ protein was confirmed on EPC surfaces by using immunofluorescence. The expression of $\beta 2 A R$ (green) and eNOS (red) and colocalization of both receptors on EPCs (yellow) are shown. $\mathbf{d}$ Representative photographs and quantitative analyses of the $\beta 2 A R$ protein expressed on EPCs after stimulation with FENO. The results show that $\beta 2 A R$ expression was not changed in the EPCs that were stimulated by using FENO. DAPI 4,6-diamidino-2-phenylindole, Dil-ac-LDL Dil-labeled acetylated low-density lipoprotein, eNOS endothelial nitric oxide synthase, EPC endothelial progenitor cell, FENO fenoterol, FITC fluorescein isothiocyanate, KDR kinase-insert domain receptor, $V W F$ von Willebrand factor, $\beta 2 A R \beta 2$ adrenergic receptor

of transplanted EPCs on endothelium recovery, we injected PB-derived EPCs into a nude mice model in which we caused wire-denuded carotid arteries. PBS, Ad5/ $/ 2$ AR-transduced EPCs, or Ad5/EGFP-transduced EPCs were injected into nude mice through the tail vein. Notably, compared with PBS, treatment with nontransduced EPCs or with Ad5/EGPF-transduced EPCs substantially increased re-endothelialization of denuded carotid arteries in nude mice; however, injecting the Ad5/B2AR-transduced EPCs resulted in a larger reendothelialization area in the injured carotid arteries
$(82.1 \pm 4.2 \%$ versus $44.4 \pm 6.5 \%$ or $46.8 \pm 4.3 \%$ versus $26.6 \pm 7.5 \%, n=5, P<0.01$; Fig. $4 \mathrm{~b})$. To determine whether labeled EPCs were able to home and incorporate into the sites of vascular endothelium injury, each mice was injected with $1 \times 10^{6}$ CM-DiI-labeled EPCs after carotid artery injury. DiI-labeled EPCs were identified as red fluorescent cells. Data showed that there were more homing EPCs in the Ad5/ $\beta 2 \mathrm{AR}$-transduced EPC group than in the Ad5/EGFP-transduced EPC group that incorporated into the FITC-lectin-positive endothelial layer (Fig. 4c, d). 

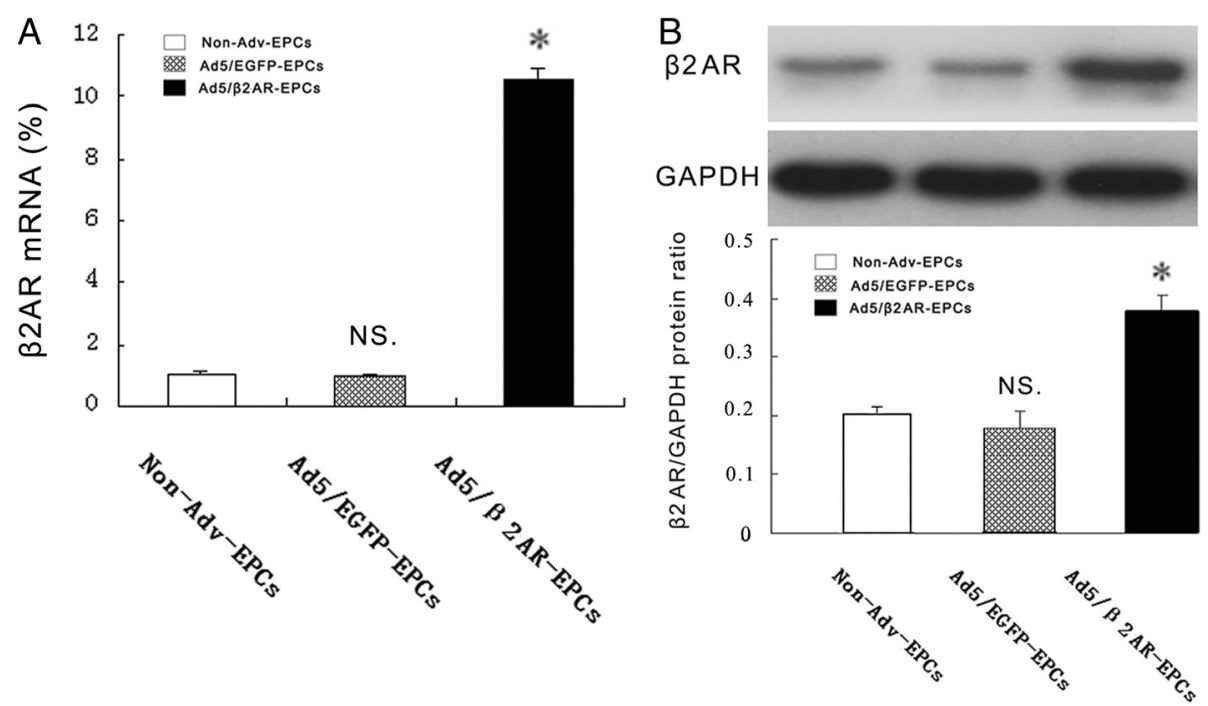

Fig. 2 Overexpression of $\beta 2 A R$ in transfected EPCs. a $\beta 2 A R$ mRNA levels were measured in non-Adv EPCs, Ad5/EGFP EPCs, and Ad5/ $\beta 2 A R$ EPCs by using real-time polymerase chain reaction and normalized against $\beta$-actin ( $n=5$ per group). ${ }^{*} P<0.01$ versus non-Adv EPCS or Ad5/EGFP EPCs. NS not significant versus non-Adv-EPCs. $\mathbf{b} \beta 2 A R$ protein levels were significantly higher in the Ad5/ $\beta 2 A R$ EPC group than in the non-Adv EPC or Ad5/EGFP-EPC group. ${ }^{*} P<0.01$ versus non-Adv EPCs or Ad5/EGFP EPCs. NS not significant versus non-Adv-EPCs ( $n=5$ per group). Ad5 adenovirus serotype 5, EGFP enhanced green fluorescent protein, EPC endothelial progenitor cell, $\beta 2 A R \beta 2$ adrenergic receptor

\section{The $\beta 2 A R / A k t / e N O S$ pathway improves EPC function in} vitro and the endothelial repair capacity of EPCs in vivo The activations of Akt and eNOS are both important to determining the number and function of active EPCs. The activation of $\beta A R s$ in cardiac progenitor cells (CPCs) was previously shown to promote the proliferation and survival of CPCs and to be associated with the increased phosphorylation of Akt and eNOS. Therefore, we examined whether the $\beta 2 A R / A k t / e N O S$ signaling pathway plays an important role in the proliferation, $\mathrm{NO}$ production, migration, and adhesion of EPCs. We used inhibitors, including ICI118,551 (a selective inhibitor of B2AR), LY294002 (an Akt inhibitor), and L-NAME (an eNOS inhibitor), to inhibit the activation of the $\beta 2 A R /$ Akt/eNOS signaling pathway.

Following stimulation with FENO $\left(10^{-8} \mathrm{M}\right)$ for $6 \mathrm{~h}$, the phosphorylation levels of Akt and eNOS were enhanced in the Ad5/ $\beta 2 A R$-transduced EPCs compared with those in the Ad5/EGFP-transduced EPCs and in the non-transduced EPCs. Furthermore, the increases in Akt and eNOS phosphorylation that were observed in the Ad5/ß2AR-transduced EPCs were inhibited by preincubation for $1 \mathrm{~h}$ with $0.3 \mu \mathrm{M}$ of ICI118,551, $10 \mu \mathrm{M}$ LY294002 or $100 \mu \mathrm{M}$ L-NAME (Fig. 5a, b). Interestingly, we also found that $\beta 2 \mathrm{AR}$ overexpression-mediated effects on proliferation, NO production, migration, and adhesion were all significantly inhibited by pretreatment with ICI118,151, LY294002, and L-NAME (Fig. 5c-f). These results indicate that the $32 \mathrm{AR} / \mathrm{Akt} /$ eNOS signaling pathway is at least partially responsible for the function of EPCs in vitro. We also investigated whether the $\beta 2 A R / A k t / e N O S$ signaling pathway is associated with the re-endothelialization capacity of EPCs in vivo. In agreement with the in vitro results, pretreatment with inhibitors of $\beta 2 \mathrm{AR}$, Akt, and eNOS attenuated the enhanced re-endothelialization in the mice that were transplanted with Ad5/ $\beta 2 \mathrm{AR}$-transduced EPCs (Fig. 5g).

\section{Discussion}

The present study demonstrates that $\beta 2 A R$ expression on EPCs is involved in the restoration of damaged endothelium. Ad5/ $\beta 2 A R$ gene transfer treatment enhanced $\beta 2 A R$ expression in EPCs and increased the capacity of EPCs to migrate, adhere, proliferate, and secrete $\mathrm{NO}$ in vitro and the re-endothelialization capacity of EPCs in vivo. Moreover, the increase in re-endothelialization capacity of EPCs is closely correlated with upregulation of the $\beta 2 A R / A k t / e N O S$ pathway. Our present study is the first to demonstrate that $\beta 2 A R s$ play a crucial role in regulating the vascular endothelial repair function of EPCs. At the molecular level, these effects were found to be at least partially associated with $\beta 2 \mathrm{AR} / \mathrm{Akt} / \mathrm{eNOS}$ pathway activation.

Endothelial dysfunction is known to play pivotal roles in degenerative vascular disease. Restoration of endothelial integrity is an important technique that can be used to cure vascular disease. Since Asahara et al. [19] first cultivated EPCs, numerous experiments have shown that EPCs mediated endothelial restoration in many processes, suggesting that they may also be useful for treating vascular injury. The term EPCs has been applied interchangeably 


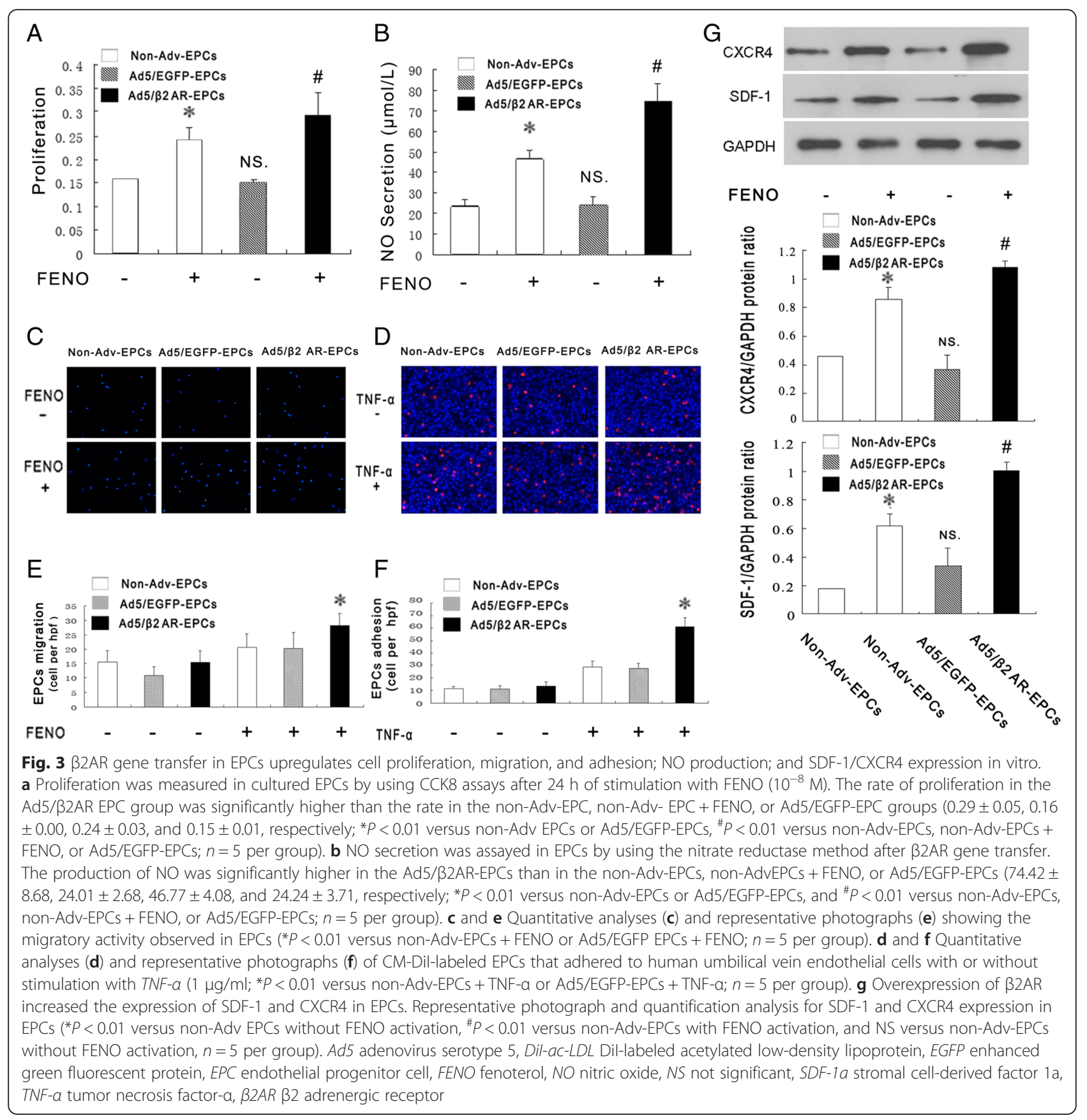

to a variety of cell populations by different investigators, suggesting that EPCs are not a single type of cell population. The definition of EPCs remains controversial. Many studies have attempted to identify cell surface markers that are unique to EPCs and to distinguish them from mature ECs and from myeloid-monocytic cells; however, these attempts have met with little success. Broadly speaking, two approaches to identify EPCs have predominated: (1) identification of cells bearing surface markers that indicate both cellular naïveté and endothelial origin and (2) inference of the presence of endothelial precursors with a given cell population by the identification of cells bearing mature endothelial characteristics after a period of culture under angiogenic conditions [20]. In our experiment, according to the method of Hur et al. [14], isolated MNCs were resuspended by using the EGM-2 BulletKit system. After 5 to 7 days, attached cells were elongated and had a spindle shape. The recognition of cell surface antigen markers by flow cytometric analysis has confirmed the cells as early EPCs, consistent with the results of other studies $[12,21]$. 

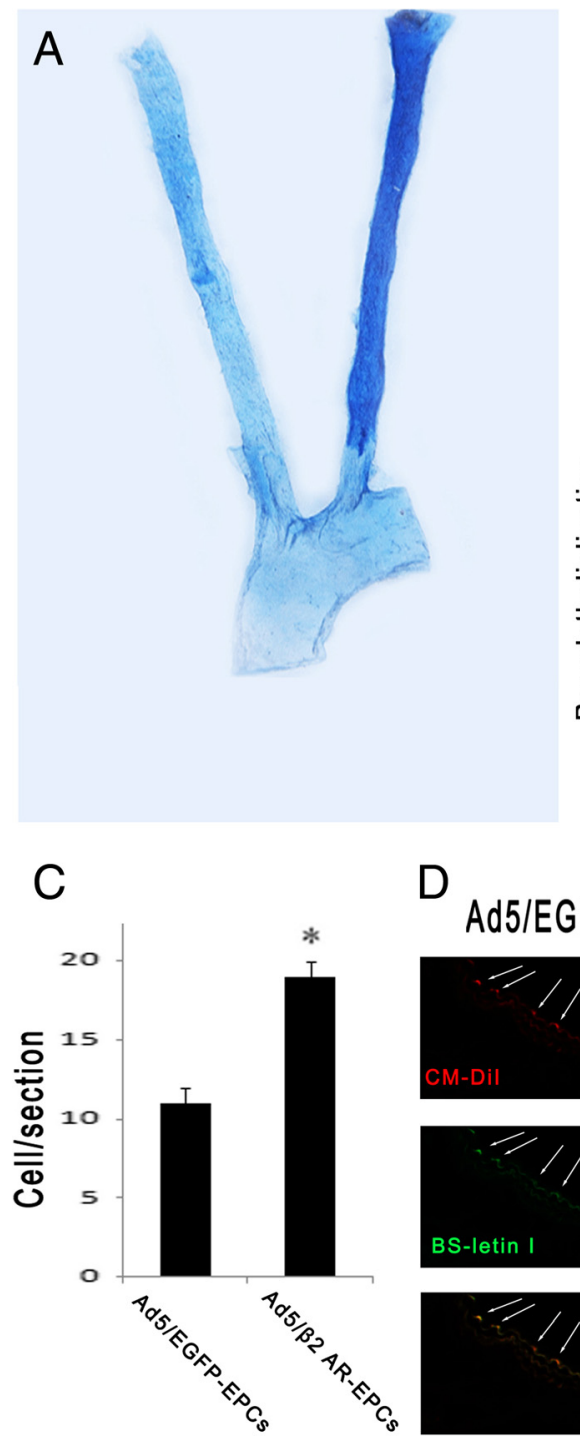
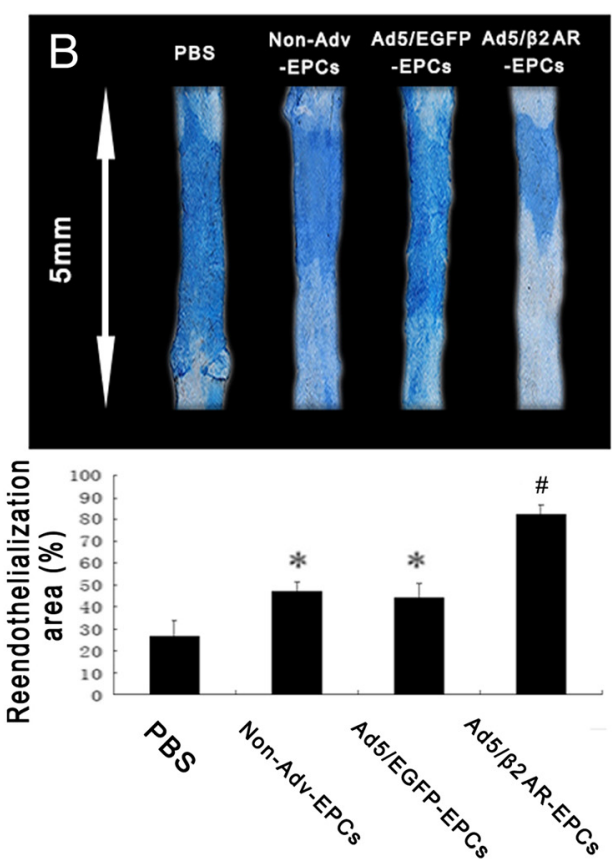

Ad5/EGFP-EPCS

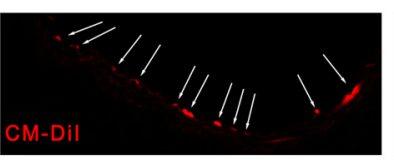

Ad5/B2AR-EPCs
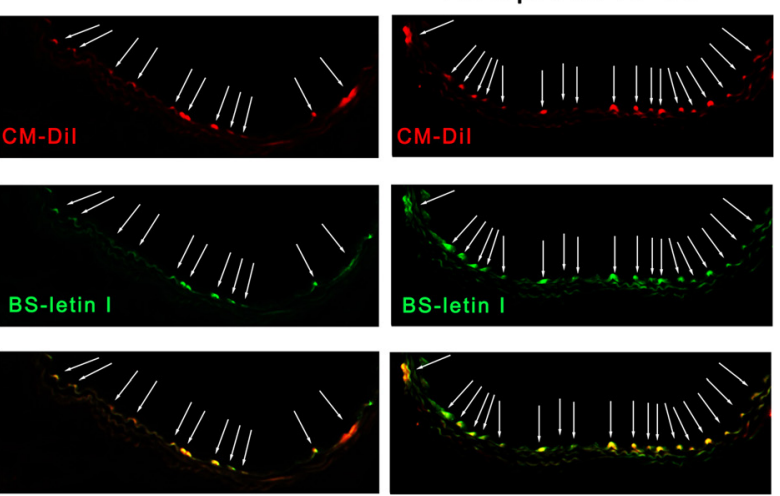

Fig. 4 B2AR gene transfer increases the re-endothelialization capacity of EPCs in vivo. Ad5/ $32 A R$ gene transfer contributed to the re-endothelialization of injured carotid arteries. a Evans blue staining was used to identify segments of denuded endothelium immediately after nude mice were subjected to wire-mediated carotid artery injury. Representative photograph shows an injured artery and a contralateral uninjured artery. $\mathbf{b}$ The transplantation of EPCs that underwent Ad5/B2AR gene transfer resulted in a higher area of re-endothelialization than was observed in the PBS-injected and non-Adv EPC transplantation group $(n=5)$ or the Ad5/EGFP EPC group $(n=5)\left({ }^{*} P<0.01\right.$ versus the PBS group, ${ }^{\#} P<0.01$ versus non-Adv-EPCs, or Ad5/EGFP-EPC group). $\mathbf{c}$ Higher numbers of homed Ad5/ $\beta 2$ AR EPCs than Ad5/EGFP EPCs were detected; $n=5$ per group $(* P<0.01$ versus Ad5/EGFP-EPC group).

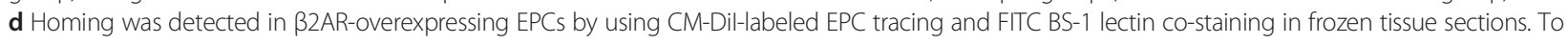
demonstrate that the transfused EPCs that localized to the injured site were endothelial cells, null mice received FITC-labeled BS-1 lectin 30 min before tissues were harvested. Ad5 adenovirus serotype 5, Dil-ac-LDL Dil-labeled acetylated low-density lipoprotein, EGFP enhanced green fluorescent protein, EPC endothelial progenitor cell, FITC fluorescein isothiocyanate, PBS phosphate-buffered saline, $\beta 2 A R \beta 2$ adrenergic receptor

Maintenance of the normal number and function of EPCs in the systemic circulation is now known to be an important novel endogenous vascular repair factor. In animal and clinical research, transplanted EPCs can home to impaired arteries, promoting re-endothelialization and reducing neointima formation. Various molecules may be involved in the process of EPC homing. EPCs and ECs have previously been shown to express both $\beta 1$ ARs and $\beta 2$ ARs $[8,9]$. $\beta 2 A$ Rs are $G$ protein-coupled receptors that can induce cell proliferation and promote cell survival in many tissues, and stimulation of endothelial $\beta 2 A R s$ has been shown to activate eNOS and release of $\mathrm{NO}$ in human umbilical vein endothelium [22]. Previous studies have shown that EPCs and ECs harvested from $\beta 2 \mathrm{AR}-$ knockout $(\beta 2 \mathrm{AR}-\mathrm{KO})$ mice are impaired in their abilities to migrate and stimulate network formation on Matrigel in vitro $[8,9]$. Moreover, when $\beta 2 A R-K O E C s$ and EPCs were injected 


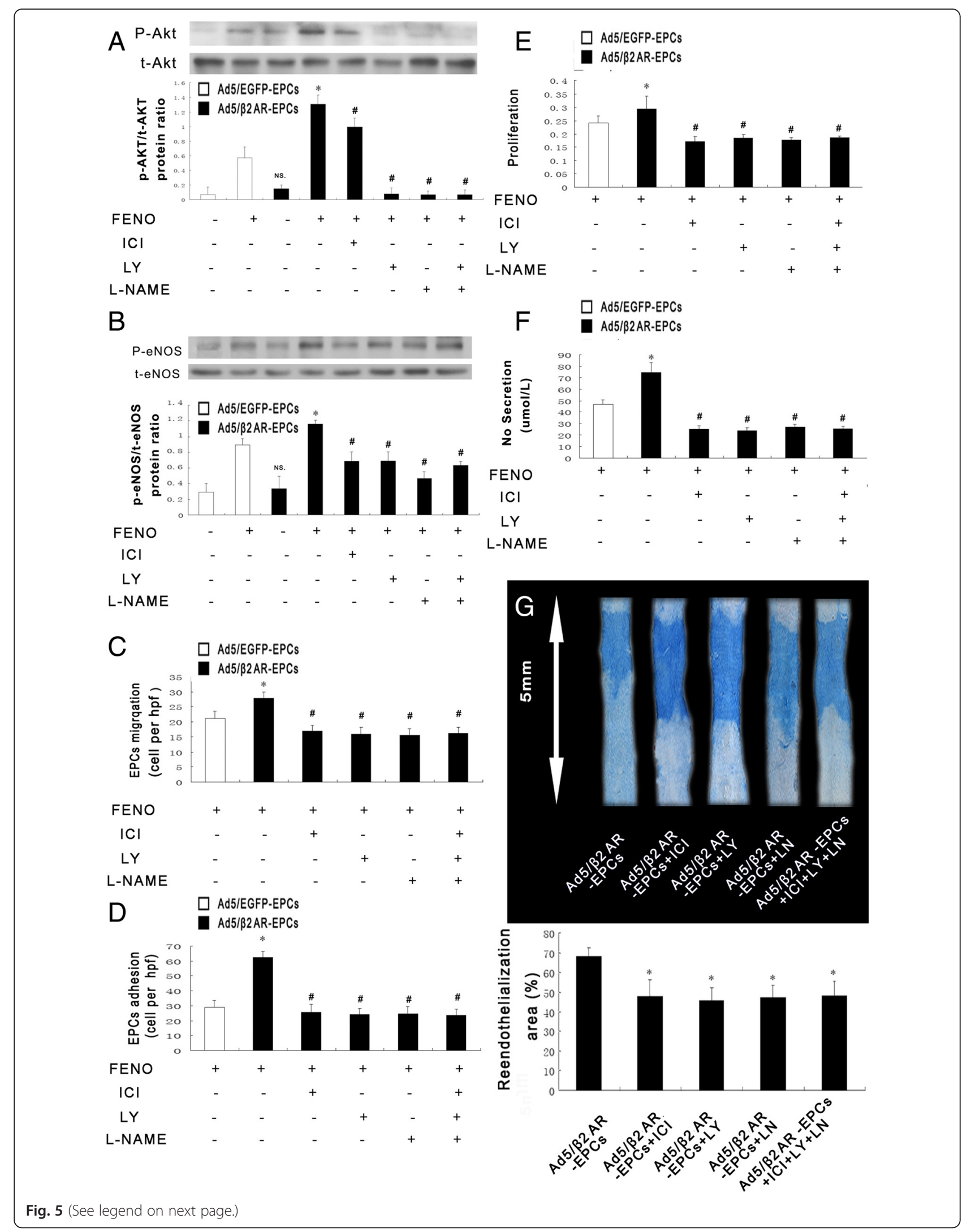




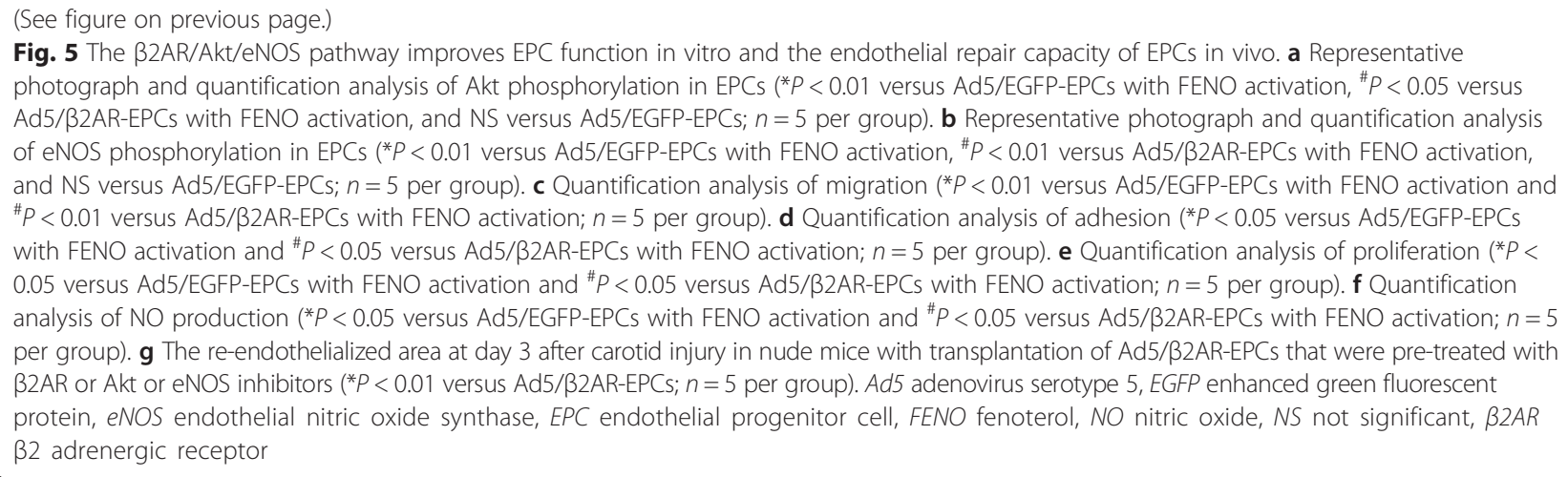

into ischemic hind limbs, no significant amelioration of neovascularization was noted [8, 9]. However, whether $32 \mathrm{ARs}$ mediate the capacity of EPCs to reendothelialize damaged arteries following an injury has never previously been tested. Given the results described in prior reports, we hypothesized that $\beta 2 A R$ overexpression in human EPCs would result in the functional enhancement of EPCs in vitro and that $\beta 2 \mathrm{AR}$ gene upregulation would contribute to the restoration of endothelial injury in vivo.

To address these assumptions, we first investigated the effect of $\beta 2 \mathrm{AR}$ overexpression on the proliferation, migration, adhesion, and NO secretion of EPCs. We found that when EPCs were stimulated with FENO, the rates of proliferation and migration were significantly higher in EPCs that overexpressed $\beta 2 \mathrm{ARs}$, indicating that FENO-induced functions were enhanced by $\beta 2 \mathrm{AR}$ overexpression in EPCs. Furthermore, our results showed that TNF- $\alpha$-induced adhesion and NO secretion were enhanced by $\beta 2 A R$ overexpression in EPCs. Given the close association between $\beta 2 A R$ and EPC function, deeper insight into the contribution of $\beta 2 A R$ expression in EPCs to accelerated reendothelialization may be of clinical importance for the treatment of CVDs. We investigated the effect of infusing cultured PB-derived EPCs in a previously described nude mouse model of carotid artery injury. As shown in Fig. 4b, $\beta 2 A R$ overexpression in the infused EPCs resulted in a significantly larger area of re-endothelialization, suggesting that $\beta 2 \mathrm{AR}$ signaling might be an important molecular mechanism that contributes to enhanced reendothelialization by EPCs. Notably, the SDF-1 $\alpha / C X C R 4$ interaction plays an important role in the regulation of a variety of cellular functions, including cell migration, proliferation, survival, and angiogenesis. Previous studies have demonstrated that the SDF-1a/CXCR4 axis is crucial for the therapeutic integrity of EPCs and their ability to home to injured vessels. For example, investigators showed that SDF-1a was involved in ischemia-mediated mobilization and homing in EPCs after vascular injury [18]. It has also been reported that CXCR4 expression restored the functions of EPCs in hypertensive patients [23]. Our data showed that stimulation with $\beta 2 A R$ promoted EPC function and significantly increased SDF-1a and CXCR4 expression in cultured EPCs, especially in EPCs that overexpressed $\beta 2 \mathrm{AR}$. These data collectively suggest that $\beta 2 \mathrm{AR}$ is an important potential therapeutic target and that B2AR-mediated EPC functions are dependent, at least in part, on the production of SDF-1a/CXCR4 and may otherwise be equal to the SDF/CXCR4 axis in the biology of EPCs. Therefore, our results indicate that transplantation of EPCs that have enhanced $\beta 2 A R$ expression could be used as a novel therapy for treating vascular endothelial injuries.

In regard to how $\beta 2 A R$ gene transfer modulates reendothelialization, several lines of evidence have shown that activation of $\beta 2 A$ Rs promoted the proliferation and survival of cardiac progenitor cells in association with increased eNOS phosphorylation [24, 25]. Akt, a multifunctional regulator of cell survival, is a downstream effector of phosphatidylinositol-3 kinase (PI3K). It is widely accepted that activating Akt phosphorylation stimulates eNOS phosphorylation at Ser1177 and increases endothelial NO production, which is involved in the mobilization of stem and progenitor cells [26]. Our current data demonstrate that this signal paradigm also exists in EPCs because $32 \mathrm{AR}$ stimulation increased the phosphorylation of Akt and eNOS, which is consistent with increased EPC proliferation, migration, and adhesion function in vitro and with accelerated re-endothelialization in vivo. These cellular effects are blocked by $\beta 2 A R / A k t / e N O S$ inhibition. Furthermore, we upregulated the expression of $\beta 2 \mathrm{AR}$ via $\mathrm{Ad} 5 / \beta 2 \mathrm{AR}$ gene transfer and found that the increased re-endothelialization capacity that was induced in the cultured EPCs that overexpressed $\beta 2 A R$ was blocked by the $\beta 2 A R$ inhibitor ICI111, 181; the Akt inhibitor LY294002; and the eNOS inhibitor L-NAME. These data strongly support the hypothesis that B2AR plays a crucial role in the regulation of vascular repairs by circulating EPCs. We believe that this study is also the first to provide data indicating that mechanisms involving $\beta 2 A R$ signaling underlie the functions of EPCs in vitro and their capacity to re-endothelialize injuries in vivo. 
The present study has some limitations. First, although we used a $\beta 2 A R$ inhibitor to explore the important functions of EPCs in the vitro and in vivo experiments, we did not focus on exploring the functional impairments that were observed in the $\beta 2 \mathrm{AR}-\mathrm{KO}$ EPCs during homing to impaired arteries. Second, $\beta 2 A R$ gene transfer results in accelerated re-endothelialization after vascular injury, but the adenovirus-mediated $\beta 2 \mathrm{AR}$ gene overexpression should be verified to determine its clinical safety. Finally, we used a relatively simple model in nude mice to demonstrate that $\beta 2 A R$ gene transfer resulted in EPCs with enhanced repair capacities. Whether the $\beta 2 \mathrm{AR} / \mathrm{Akt} / \mathrm{eNOS}$ signaling pathway contributes more or less to EPCs to mediate endothelial repair relative to the contributions of other signaling pathways needs to be further investigated.

\section{Conclusions}

Our study is the first to provide direct evidence that $\beta 2 A R$ expression in EPCs is an important molecular target for therapeutic studies and that $\beta 2 \mathrm{AR}$ activation may be responsible for accelerated EPCs homing during injury. More importantly, our study demonstrates that overexpressing the $\beta 2 A R$ gene in EPCs results in rapid reendothelialization and largely improved post-injury vascular repairs and that these processes are mediated by the $\beta 2 \mathrm{AR} /$ Akt/eNOS signaling pathway. Stem cell therapies have shown great potential as strategies aimed at increasing the efficiency of vascular regeneration; therefore, $\beta 2 \mathrm{AR}$ gene may become a novel therapeutic molecular target in clinical studies aimed at improving cardiovascular care. These scientific questions deserve further investigation.

\section{Abbreviations}

Ad5: adenovirus serotype 5; BSA: bovine serum albumin; CVD: cardiovascular disease; DAPI: 4',6-diamidino-2-phenylindole; Dil-ac-LDL: Dil-labeled acetylated low-density lipoprotein; EBM-2: endothelial basal medium-2; EC: endothelial cell; EGFP: enhanced green fluorescent protein; eNOS: endothelial nitric oxide synthase; EPC: endothelial progenitor cell; FBS: fetal bovine serum; FENO: fenoterol; FITC: fluorescein isothiocyanate; GAPDH: glyceraldehyde 3-phosphate dehydrogenase; HUVEC: human umbilical vein endothelial cell; ICl: ICl118,551 (a selective inhibitor of $\beta 2 A R$ ); KDR: kinase-insert domain receptor; KO: knockout; LY: LY294002 (an Akt inhibitor); mAb: monoclonal antibody; MNC: mononuclear cell; MOl: multiple of infection; NO: nitric oxide; PB: peripheral blood; PBS: phosphate-buffered saline; PCR: polymerase chain reaction; PE: phycoerythrin;

PFA: paraformaldehyde; SDF-1a: stromal cell-derived factor 1a; TNF-a: tumor necrosis factor-a; VEGF: vascular endothelial growth factor; VWF: von Willebrand factor; $\beta 2$ AR: $\beta 2$ adrenergic receptor.
}

\section{Competing interests}

The authors declare that they have no competing interests.

\section{Authors' contributions}

XK and R-QN designed and conducted experiments, analyzed the data, and compiled the main manuscript. X-RS and FW conducted immunohistochemical experiments and evaluated enzyme activities. Q-SH and B-QD conducted animal experiments. J-FW conducted physiological measurements and prepared samples. All authors read and approved the final manuscript.

\section{Acknowledgements}

This study was supported by a grant from the National Natural Science Foundation of China (NO.81370309).

\section{Author details}

'Department of Cardiology, Sun Yat-sen Memorial Hospital of Sun Yat-sen University, No. 107, Yanjiangxi Road, Guangzhou, China. ${ }^{2}$ Guangdong Province Key Laboratory of Arrhythmia and Electrophysiology, Guangzhou 510120, China. ${ }^{3}$ Department of Geriatric, The First Affiliated Hospital of Sun Yat-sen University, Guangzhou 510080, China.

Received: 5 March 2016 Revised: 27 April 2016

Accepted: 4 May 2016 Published online: 18 May 2016

\section{References}

1. Sen T, Aksu T. Endothelial progenitor cell and adhesion molecules determine the quality of the coronary collateral circulation/endothelial progenitor cells (CD34 + KDR+) and monocytes may provide the development of good coronary collaterals despite the vascular risk factors and extensive atherosclerosis. Anadolu Kardiyol Derg. 2012;12:447-8.

2. Mannarino E, Pirro M. Endothelial injury and repair: a novel theory for atherosclerosis. Angiology. 2008;59:69S-72.

3. Inoue T, Croce K, Morooka T, Sakuma M, Node K, Simon DI. Vascular inflammation and repair: implications for re-endothelialization, restenosis, and stent thrombosis. JACC Cardiovasc Interv. 2011;4:1057-66.

4. Yin Y, Liu H, Wang F, Li L, Deng M, Huang L, et al. Transplantation of cryopreserved human umbilical cord blood-derived endothelial progenitor cells induces recovery of carotid artery injury in nude rats. Stem Cell Res Ther. 2015;6:37.

5. Zhu S, Malhotra A, Zhang L, Deng S, Zhang T, Freedman NJ, et al. Human umbilical cord blood endothelial progenitor cells decrease vein graft neointimal hyperplasia in SCID mice. Atherosclerosis. 2010;212:63-9.

6. Li X, Chen C, Wei L, Li Q, Niu X, Xu Y, et al. Exosomes derived from endothelial progenitor cells attenuate vascular repair and accelerate reendothelialization by enhancing endothelial function. Cytotherapy. 2016;18:253-62.

7. Alexandru N, Popov D, Dragan E, Andrei E, Georgescu A. Circulating endothelial progenitor cell and platelet microparticle impact on platelet activation in hypertension associated with hypercholesterolemia. PLoS One. 2013;8:e52058.

8. laccarino G, Ciccarelli M, Sorriento D, Galasso G, Campanile A, Santulli G, et al. Ischemic neoangiogenesis enhanced by beta2-adrenergic receptor overexpression: a novel role for the endothelial adrenergic system. Circ Res. 2005:97:1182-9.

9. Galasso G, De Rosa R, Ciccarelli M, Sorriento D, Del GC, Strisciuglio T, et al. $\beta 2$-adrenergic receptor stimulation improves endothelial progenitor cellmediated ischemic neoangiogenesis. Circ Res. 2013;112:1026-34.

10. Hill JM, Zalos G, Halcox JP, Schenke WH, Waclawiw MA, Quyyumi AA, et al. Circulating endothelial progenitor cells, vascular function, and cardiovascular risk. N Engl J Med. 2003:348:593-600.

11. Hristov M, Erl W, Weber PC. Endothelial progenitor cells: mobilization, differentiation, and homing. Arterioscler Thromb Vasc Biol. 2003;23:1185-9.

12. Chen L, Wu F, Xia WH, Zhang YY, Xu SY, Cheng F, et al. CXCR4 gene transfer contributes to in vivo reendothelialization capacity of endothelial progenitor cells. Cardiovasc Res. 2010;88:462-70.

13. Zhang XY, Su C, Cao Z, Xu SY, Xia WH, Xie WL, et al. CXCR7 upregulation is required for early endothelial progenitor cell-mediated endothelial repair in patients with hypertension. Hypertension. 2014;63:383-9.

14. Hur J, Yoon $\mathrm{CH}$, Kim HS, Choi JH, Kang HJ, Hwang KK, et al. Characterization of two types of endothelial progenitor cells and their different contributions to neovasculogenesis. Arterioscler Thromb Vasc Biol. 2004;24:288-93.

15. Giannotti G, Doerries C, Mocharla PS, Mueller MF, Bahlmann FH, Horvàth T, et al. Impaired endothelial repair capacity of early endothelial progenitor cells in prehypertension: relation to endothelial dysfunction. Hypertens. 2010;55:1389-97.

16. Nishiwaki Y, Yoshida M, Iwaguro H, Masuda H, Nitta N, Asahara T, et al. Endothelial E-selectin potentiates neovascularization via endothelial progenitor cell-dependent and -independent mechanisms. Arterioscler Thromb Vasc Biol. 2007;27:512-8.

17. Walter DH, Haendeler J, Reinhold J, Rochwalsky U, Seeger F, Honold J, et al. Impaired CXCR4 signaling contributes to the reduced neovascularization capacity of endothelial progenitor cells from patients with coronary artery disease. Circ Res. 2005;97:1142-51. 
18. Yin $Y$, Zhao $X$, Fang $Y$, Yu S, Zhao J, Song M, et al. SDF-1alpha involved in mobilization and recruitment of endothelial progenitor cells after arterial injury in mice. Cardiovasc Pathol. 2010;19:218-27.

19. Asahara T, Murohara T, Sullivan A, Silver M, van der Zee $R, L i T$, et al. Isolation of putative progenitor endothelial cells for angiogenesis. Science. 1997;275:964-7.

20. Padfield GJ, Newby DE, Mills NL. Understanding the role of endothelial progenitor cells in percutaneous coronary intervention. J Am Coll Cardiol. 2010;55:1553-65.

21. Xia WH, Yang Z, Xu SY, Chen L, Zhang XY, Li J, et al. Age-related decline in reendothelialization capacity of human endothelial progenitor cells is restored by shear stress. Hypertension. 2012;59:1225-31.

22. Xu B, Li J, Gao L, Ferro A. Nitric oxide-dependent vasodilatation of rabbit femoral artery by beta(2)-adrenergic stimulation or cyclic AMP elevation in vivo. Br J Pharmacol. 2000;129:969-74.

23. Liu X, Zhang GX, Zhang XY, Xia WH, Yang Z, Su C, et al. Lacidipine improves endothelial repair capacity of endothelial progenitor cells from patients with essential hypertension. Int J Cardiol. 2013;168:3317-26.

24. Duda DG, Fukumura D, Jain RK. Role of eNOS in neovascularization: NO for endothelial progenitor cells. Trends Mol Med. 2004;10:143-5.

25. Khan M, Mohsin S, Avitabile D, Siddiqi S, Nguyen J, Wallach K, et al. $\beta$ adrenergic regulation of cardiac progenitor cell death versus survival and proliferation. Circ Res. 2013;112:476-86.

26. Aicher A, Heeschen C, Mildner-Rihm C, Urbich C, Ihling C, Technau-Ihling K, et al. Essential role of endothelial nitric oxide synthase for mobilization of stem and progenitor cells. Nat Med. 2003;9:1370-6.

\section{Submit your next manuscript to BioMed Central and we will help you at every step:}

- We accept pre-submission inquiries

- Our selector tool helps you to find the most relevant journal

- We provide round the clock customer support

- Convenient online submission

- Thorough peer review

- Inclusion in PubMed and all major indexing services

- Maximum visibility for your research

Submit your manuscript at www.biomedcentral.com/submit

C Biomed Central 